Pecvnia, 8 (2009), pp. 71-96

\title{
Un Manual de Aritmética mercantil de Mosén Juan de Andrés
}

Recibido: Diciembre 2008

Aceptado: Septiembre 2009
Betsabé Caunedo del Potro

betsabe.caunedo@uam.es

Universidad Autónoma de Madrid

Fac. de Filosofía y Letras

Carretera de Colmenar, Km.15

28049 Madrid (España)
La difusión de aritméticas mercantiles por Europa Occidental en los últimos siglos de la Edad Media, debemos entenderlos como una lógica consecuencia de la expansión y desarrollo del comercio. La evolución de las técnicas mercantiles convirtió a esta disciplina en una herramienta de apoyo y de uso imprescindible para el mercader, quien llegó incluso a considerarla como razón clave del éxito empresarial. Esta alta consideración estimuló su aprendizaje y la difusión de manuales como el
The spreading of arithmetic for merchants in Western Europe in the last centuries of the Middle Ages, should be understand as logical consequence of the expansion and development of trade. The evolution of the mercantile techniques turned this knowledge into an indispensable tool of support of use by the merchant, who even considered it the key of success in trading. This high opinion on it was strong stimulus to learn it and the cause or the wide diffusion of Manual like one presented

* Este trabajo ha sido elaborado en el marco del proyecto de investigación HUM 2007-63856, La transmisión del saber técnico y profesional: literatura técnica en la España Medieval, subvencionado por el Ministerio de Ciencia y Tecnología. 
que aquí presentamos, elaborado ya en los primeros años del siglo XVI.

Palabras clave: Aritmética mercantil, desarrollo del comercio, aprendizaje mercantil, manuales bajomedievales y renacentistas. here, produced in the early years of the XVI th century.

Key words: Arithmetic for merchants, development of trade, learning mercantile techniques, Manual from the Lower Middle Ages and Renaissance.

El objeto de este trabajo es hacer una breve aproximación al manual de aritmética mercantil elaborado por Mosén Juan de Andrés en los albores del siglo XVI, Sumario breve de la practica de la Aritméthica. $Y$ lo hago apuntando una primera consideración sobre el mismo que me satisface especialmente dada mi condición de medievalista y es la enorme deuda que éste y otros textos de los primeros años del siglo XVI tienen con las aritméticas medievales. Éstas, revolucionarias en su día, las debemos contemplar hoy, como testigos activos y brillantes del dinamismo y eficacia de unos hombres de negocios y de la pujanza urbana a ellos ligada. Efectivamente, toda la producción aritmética occidental de los siglos XIII, XIV y XV aparece íntimamente ligada a la "revolución comercial" y como herramienta de apoyo imprescindible de unas actividades contables y fiscales.

Las técnicas mercantiles habían ido evolucionando desde el siglo XI. Primero lentamente, después a mayor velocidad, se había ido recorriendo un largo camino de progreso y desarrollo, no exento de tropiezos, que permitió superar la excepcionalidad e irregularidad del incipiente comercio de esos años. La definitiva sedentarización del mercader, la profesionalidad del servicio de transporte, las nuevas formas de asociaciones mercantiles y de aportación de capital, el mayor uso de los sistemas de crédito, los nuevos modos de llevar la contabilidad... exigía al mercader moverse con soltura en un mundo cada vez más complejo y diversificado que suponía tanto conocer mercados y rutas diferentes como instrumentos de cambio, medios de transporte o reglas aritméticas con las que efectuar diferentes operaciones y llevar con eficacia su nueva contabilidad. Igualmente el carácter internacional de muchas transacciones y la ya comentada sedentarización del mercader, cabeza de una compañía y gestor responsable ante sus "compañeros" de los negocios que capitaneaba, requerían una correspondencia mercantil y una red de información, totalmente necesaria para asegurarse el éxito y/o desviar la competencia. Además, problemas como los endeudamientos 
de las monarquías, las pérdidas de créditos, las quiebras de bancos, las alteraciones monetarias... sufridas durante la denominada "crisis del siglo XIV"1 habían alertado al mercader. En ese ambiente, un aprendizaje determinado empezaba a perfilarse como razón del éxito empresarial a la vez que se consideraba imprescindible ${ }^{2}$.

Ese aprendizaje -no exclusivamente empírico- y que cubriría una serie de etapas ${ }^{3}$, se iniciaba como cualquier otro con la enseñanza de la lectura, la escritura y el cálculo. Debemos destacar que estas disciplinas se utilizaron como herramientas de trabajo, no valorándose en sí mismas, sino como instrumentos que permitían y facilitaban una mejor administración de negocios y propiedades. Se extendió así un alfabetismo de cuño utilitario, práctico y profesional ${ }^{4}$ que junto con el cálculo constituyó un conocimiento indispensable para llevar a buen término cualquier negocio mercantil. $Y$ aunque existía un abismo entre el comercio internacional a gran escala y el comercio al detalle de algunos mercaderes, a todos los que practicaban cada una de sus modalidades, al igual que a otros muchos sectores urbanos, les interesó el desarrollo de una aritmética práctica ${ }^{5}$. Unos y otros, sintiendo esa imperiosa necesidad de formación, manifestaron un cierto culto a las cifras, al manejo correcto de las mismas, a las proporciones, a la exactitud..., propiciando un nuevo sistema de valores que lentamente acabaría imponiéndose. No tenemos más que fijarnos en Barcelona: Crítica, pp. 153-159.

C. Cipolla (1994) El gobierno de la moneda. Ensayos de historia monetaria,

2 Hilario Casado considera que la creación de instituciones y técnicas que permitieron que las compañías castellanas redujesen costes y fuesen más eficaces y competitivas favoreció el éxito y prosperidad del comercio castellano a fines de la Edad Media. H. Casado Alonso (1999) "Comercio y nacimiento del Estado Moderno en Castilla (S. XIV - XVI). Algunas reflexiones a la luz de nuevas corrientes de investigación internacional", El Estado en la Baja Edad Media. Nuevas perspectivas metodológicas. Universidad de Zaragoza, pp. 51-75.

3 Ver al respecto mi trabajo (2006) "La formación y educación del mercader", $E l$ Comercio en la Edad Media, XVI Semana de Estudios Medievales Nájera y Tricio 2005, Nájera, pp. 417-454.

4 Según la expresión del prof. Castillo Gómez (1997) De Escrituras y Escribientes. Práctica de la cultura escrita en una ciudad del Renacimiento. Las Palmas de Gran Canaria: Fundación de Enseñanza Superior a distancia, p. 271. Explica como se trataba de una alfabetización restringida en la que el aprendizaje "no es un fin en si mismo, sino consecuencia de un uso necesario o directamente útil".

Pensemos, por ejemplo, en las necesidades fiscales del nuevo estado o de los diferentes municipios. Contar a la población y el dinero serán instrumentos básicos para su actuación. Colocar a los jóvenes en situación de dominar las técnicas de la profesión a la que se dedicarían en un futuro próximo, fue un objetivo a conseguir por diferentes sectores urbanos. Mercaderes de diferente nivel, artesanos, arrendadores de impuestos... forman parte de un grupo que se amplía continuamente entre los siglos XIII y XV y que se perfila como demandadores de una formación específica. Ver sobre esta problemática, B. Caunedo del Potro y R. Córdoba de la Llave (2004) "Oficios Urbanos y desarrollo de la Ciencia y de la Técnica en la Baja Edad media: La Corona de Castilla", Norba, Revista de Historia, 17, pp. 41-68. 
las cualidades y virtudes que pregonaban los Manuales medievales como necesarias para ser un buen mercader para entenderlo. El gusto por el orden, la claridad, la precisión... también la discreción, la lealtad, la honorabilidad o la prudencia se ensalzan continuamente. Dejaban claro que la confianza en el mundo comercial se ganaba cada día mediante la práctica permanente y sin excepción de valores éticos como la honestidad, confidencialidad, lealtad y honorabilidad. El profesionalismo se entendía ya como la prestación oportuna de servicios de calidad basados en la experiencia, el conocimiento y la prudencia ${ }^{6}$.

En este marco de crecimiento, en el que tomar ventaja requería capacitarse en nuevas habilidades, se difundieron desde Italia, a raíz de su iniciativa, todas las innovaciones técnicas. Entre ellas, sus logros en el campo del aprendizaje y formación mercantil. En este país encontramos las mejores escuelas de ábaco ${ }^{7}$, centros de formación celosamente construidos y mantenidos por las grandes ciudades italianas y a las que diariamente acudían sus jóvenes, futuros mercaderes. Y encontramos también, los ejemplos más numerosos, sino primeros ${ }^{8}$, de

6 En algunos de los Manuales de Mercaduria a los que nos referiremos inmediatamente, se dedican unas líneas a la descripción de las cualidades de un buen mercader. Un ejemplo es el Llibre de coneixences d'especies $i$ de drogues $i$ d'avisaments de pesos, canes $i$ mesures di diverses terres y del que M. Gual Camarena (1981) El primer Manual Hispánico de Mercaderia (siglo XIV), Barcelona, realiza una buena edición crítica. Para su anónimo autor, el mercader ideal debe gozar de buena salud, ser justo, sabio, discreto, tener don de gentes y además reunir las cualidades de discreción, veracidad, lealtad, diligencia, sobriedad, templanza, religiosidad... cualidades de las que también debe participar el mercader ideal descrito por el más tardio manual mallorquín Primera faç d'art de mercaderia o el Llibre que explica el que ha de ser un bon mercader, que insistiendo en este tipo de cuestiones se acerca al Libri di Buoni Customi, del florentino Paolo de Certaldo, libro de filosofía moral y consejos comerciales y que se considera como el más importante de este tipo de tratados, diferentes de los Manuales de Mercaderia y del que A. De Schiaffani, ya en 1945, realizó una edición. P. Certaldo (1945) Libri di Buoni Customi, ed. A. De Schiaffani, Florencia.

Al manual mallorquín se han acercado F. Sevillano Colón (1974-79) "Un Manual mallorquín de mercaduria medieval", Anuario de Estudios Medievales, 9, pp. 517-530 y A. Santamaría Arández (1993) "La compilación mallorquina de mercaderia de Domingo Pau", Homenatge a Antoni Mut Calafell, arxiver, Mallorca, pp. 249-276. De todos los escritos en lengua catalana nos ofrece un breve resumen, L. Cifuentes i Comamala (2001) La Ciencia en cátala a l'Edad Mitjana $i$ el Renaixement, Barcelona, Palma de Mallorca, pp. 310-315.

7 Ver las páginas que les dedico en (2006) "La formación y educación del mercader", El Comercio en la Edad Media, XVI Semana de Estudios Medievales Nájera y Tricio 2005 Nájera, pp. 436-439.

Recordemos que además de la introducción a gran escala del sistema numeral indio y del sistema posicional en base 10, los musulmanes volvieron a dar a las matemáticas una finalidad práctica, aplicándola a situaciones concretas de la vida diaria. Tipificaron un género y una denominación, aritmética mercantil, cuyos ejemplos nos mostrarían sus Liber Mahameleth. Algunos ejemplos de éstos podemos encontrar en la Península Ibérica. Ver al respecto mi trabajo "La formación y educación..., pp. 429-431. En esta última incluimos los comentarios de J. Hoyrup sobre el primer escrito en lengua vulgar elaborado en Umbria a finales del siglo XIII, y que considera mucho más que una vernaculización de Fibonacci, ya que de sus 31 capítulos en al menos 23 no toma préstamos del Liber Abaci, inspirándose en otras fuentes. El Liber Abaci de Leonardo Pisano escrito 
diferentes tipos de manuales de los que ya hemos hecho mención: Los Manuales de Mercaduría y los de Aritmética Práctica, a los que nos acercaremos más al presentar uno de ellos, el de Mosén Juan de Andrés. Unos y otros se destinaron a la formación mercantil, pero su uso resultó muy diferente. Mientras que los manuales de aritmética práctica eran textos considerados escolares -muchos de los maestros de ábaco italianos elaboraban sus propios textos para utilizarlos en sus escuelas-, los de Mercaduría contenían conocimientos sistematizados a partir de experiencias vividas en la organización. Se trataba de una información restringida y privada. Ésta, muy variada -pesos, medidas, peajes, tasas, características de los productos, rutas, itinerarios...- eran fruto de la experiencia acumulada por los miembros de una compañía, y sólo a ellos se dirigía. Las firmas, con su elaboración facilitaban la transmisión y conservación de unos conocimientos considerados imprescindibles para lograr el éxito, pero que no debían trascender. Tomaban medidas para salvaguardar y proteger su información ya que su difusión podía acarrear un riesgo para la firma9.

Los Manuales de Aritmética Mercantil tuvieron un carácter más general, pues se concibieron, como hemos dicho, como textos escolares. Dada esta orientación eminentemente práctica, sus ricas colecciones de problemas reflejarían, sin lugar a dudas, situaciones concretas en las que los mercaderes podrían verse involucrados. Esta particularidad nos brinda la posibilidad de conocer diversos datos de interés económico y comercial que pueden servir para ampliar, matizar o confirmar una información ya conocida, de ahí, que con las debidas precauciones, puedan utilizarse sus datos como fuente histórica.

Los diferentes manuales medievales castellanos que $\operatorname{conocemos}^{10}$, pueden resultar más o menos ricos en este tipo de datos.

en latín abre el paso a una serie de tratados elaborados en lengua vulgar para uso fundamentalmente del grupo mercantil.

Remito a mi trabajo citado en la nota 6, pp. 449-454.

10 Elaborados durante la Edad Media, son, por ahora, cuatro los que conocemos. Tres escritos en castellano, El arte del alguarismo, manuscrito 46 de la Real Colegiata de San Isidoro de León, De Arismética, Ms. 155 de la Real Academia Española y un tercero sin título, que se conserva en la Biblioteca Nacional. Sobre estos tres hemos trabajado, B. Caunedo del Potro y R. Córdoba de la Llave (2000) El Arte del Alguarismo. Un libro castellano de aritmética comercial y de ensayo de moneda del siglo XIV, Salamanca; B. Caunedo del Potro (2003-04) "De Arismética. Un manual de aritmética para mercaderes", Cuadernos de Historia de España, LXXVIII, pp. 35-46, y "Three Castilian Manuscripts on Mercantile Arithmetic: Their problems of alloys", presentada en el Coloquio Culture monétaire, aspects mathématiques, technologiques et marchands (XIII-XVI siécle),celebrado en París y Orleans en Septiembre del 2004 (actas en prensa). Entre estos tres manuscritos existen diferencias sustanciales en cuanto a la información que de carácter comercial pueden aportar. El manuscrito 10.106, Raros, Biblioteca Nacional, se centra muy especialmente en el Mediterráneo. Ribereñas al 
Pero, en general, todos nos proporcionan una interesante información sobre diversas cotizaciones de monedas, medidas de longitud, peso y capacidad, mercancías comercializadas y sus precios, itinerarios más frecuentados, ganancias que se obtenían en su compraventa, tipos de interés aplicados en las operaciones... ajustándose en general a la realidad, pues, como hemos mencionado, los autores procuraban utilizar magnitudes obtenidas de la vida real, con objeto de ir familiarizando a los jóvenes aprendices con aquellos parámetros con los que más tarde tendrían que desenvolverse.

Tras estos manuales medievales aparece el tratado que vamos a presentar, el del clérigo zaragozano, Juan de Andrés, Sumario breve de la practica de la Aritméthica ${ }^{11}$, impreso en Valencia en el taller de Juan Joffre, uno de los más activos de la ciudad en los primeros decenios del siglo $\mathrm{XVI}^{12}$. Se trata pues de un bello post incunable según la precisa definición de Julián Martín Abad del que se ha realizado también una edición facsimilar ${ }^{13}$.Comienza con un breve prefacio en el que la invocación religiosa, que no falta en ninguno de los tratados medievales, es sustituida por la dedicatoria al noble a quién está destinado, fórmula más al uso en los nuevos tiempos. En este caso, se trata del muy ilustre y

Mediterráneo son las ciudades que en más ocasiones se repiten, mediterráneas con también las monedas y las mercancías. Venecia, Acre, Pisa, Marsella... son las ciudades y dominaciones más repetidas. Sicilia -Venecia y Acre- Venecia resultan los itinerarios y trayectos por los que circulan la mayor parte de las mercancías: aceite, seda, paños de Pisa o de Marsella, que viajan casi siempre en compañía de las valoradas especies: pimienta y clavo. Todas, se pagarán en diferentes monedas, pero los besantes, quirates, tarines, torneses y barceloneses no faltan en los textos de los diferentes ejercicios.

A estos tres textos escritos en castellano debemos añadir, uno en catalán, Summa de lárt d'Aritmética, estudiado por Antoni Malet. Es una cifra muy modesta, aunque significativa, si se compara con los más de 300 recogidos por W. van Egmond en su importante catálogo. W. van Egmond (1980) Practical Mathematics in the Italian Renaissance: A Catalog of Italian Abbacus Manuscripts and Printed Books to 1660, Firenze: Istituto e Museo di Storia della Scienza. Todos ellos proporcionan una información comercial de características similares a pesar de las posibles diferencias que acabamos de comentar observadas en los manuscritos castellanos.

11 Este tratado se cobija en la Biblioteca Nacional, sección Raros, 9124. Se imprimió en Zaragoza en 1515. En adelante citaremos solamente: B.H. Raros, 9124.

J. Martín Abad (2001) Post Incunables Ibéricos, Madrid, p. 90.

Aquí nos da noticia de su inclusión en el trabajo de Bartolomé José Gallardo en su Ensayo de una biblioteca española de libros raros y curiosos, pero nosotros tuvimos noticia del mismo a partir del trabajo de E. Hernández Esteve (1981) Contribución al estudio de la historiografía contable en España, Madrid.

12 J. Martín Abad (2003) Los primeros tiempos de la imprenta en España (c. 1471-1520), Madrid, p. 100.

13 Post incunable es el término elegido por Martín Abad para referirse a los ejemplares de las ediciones impresas en talleres de España y Portugal entre los años 1501-1520. J. Martín Abad (2001) Post Incunables Ibéricos, p. 15.

La edición facsimilar: Salamanca, Universidad; Valencia, Vicente García, 1999. (Colección de Incunables y Libros Antiguos). Va acompañada por un estudio, fundamentalmente de carácter filológico a cargo de Antonio Ferrando Francés. 
muy magnifico señor Don Seraphin, conde de Silva y señor de las villas de Nulles y Pego. Tras esta invocación, un alegato sobre el valor y la utilidad de la aritmética, una exaltación de la misma, en el que se insiste en que su conocimiento facilita el estudio y desarrollo de otras disciplinas tales como la astronomía, la música, o la medicina, da paso al plan de la obra, al contenido en sí del libro, un índice o resumen. Antes de la exposición, el clérigo zaragozano vuelve a reforzar la altísima consideración que le merece la disciplina que va a explicar con continuas alusiones a la también elevada consideración que de la misma se tenía en la Antigüedad clásica. Resultaba un buen criterio de autoridad, a la vez que las alusiones a Aristóteles, a Alejandro, al templo de Minerva o al de Mares, le permitían al autor demostrar y lucir su erudición ${ }^{14}$.

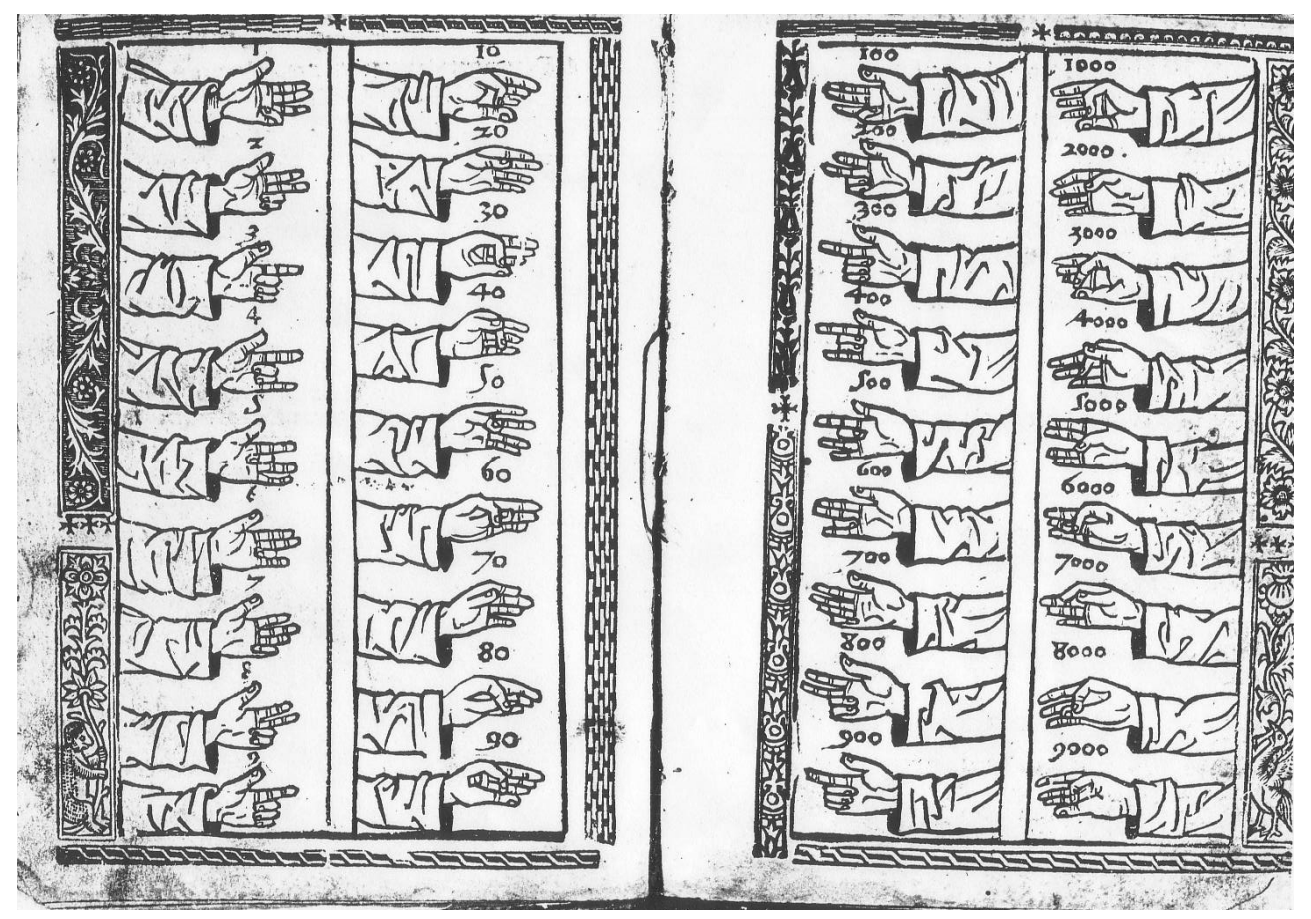

Números y "lenguaje digital" que el autor inserta en su Manual tras la dedicatoria (sin foliar). Obsérvese que se trata de la misma tabla que reproduce Luca Pacioli en Suma Aritmética, Geometría, Proportioni et Proporcionalita, publicada en Venecia en 1494. A su vez, ésta recuerda la de Beda el Venerable en De rationi temporarum aunque en el caso veneciano y en el que aquí reproducimos los "cientos" y los "miles" se cuentan con la mano derecha.

14 B.N. Raros, 9124. El manual está foliado (parte superior derecha) con numeración romana. Comienza con el tratado propiamente dicho, no numerando, por tanto, la dedicatoria ni el índice, aunque cuando inicia la numeración comienza en el folio IX. 
A continuación, presenta el trabajo, al que le ha dado un claro valor instrumental, estructurado en 10 tratados o partes, cada una de las cuales estará, a su vez, subdividido en diferentes apartados, que el autor llama capítulos y artículos ${ }^{15}$. El primer tratado, que se compone de tres capítulos, lo dedica al número y a su definición. Enumera: número par, impar, cuadrado, cúbico, perfecto, imperfecto ${ }^{16}$. En el segundo, explica las operaciones aritméticas básicas, que el autor denomina espeçias, término que también vimos emplear al autor del Arte del Alguarismo en el siglo XIV, y que son siete: nombrar, sumar, restar, multiplicar, partir, progresiones y raíces cuadradas y cúbicas ${ }^{17}$. A cada una de estas operaciones dedica un capítulo, y tras habernos explicado en la primera espeçia, nombrar, el sistema de numeración indoarábigo y el sistema en base 10, sigue siempre la misma dinámica: define someramente en qué consiste la operación, la explica, y nos expone la forma de probar si está bien hecha. En el tercer tratado, define los quebrados y nos enseña a operar con ellos y también con los números mixtos ${ }^{18}$.

A la regla de tres dedica el tratado cuarto y el quinto, a la regla de compañía ${ }^{19}$, fijándose en el sexto tratado en la regla de las baratas. Antes de explicarla y presentar sus tipos y los problemas correspondientes, las define, y así, entendemos, que una barata simple no es más que una mera operación de intercambio de mercancías de igual valor, un trueque. Una barata compuesta, responde al cambio de mercancías de valor diferente, por lo que para igualarlas, se exige además una cierta cantidad

15 B.N. Raros, 9124, "... en el qual se contienen diez tratados y cada tratado contiene ciertos capítulos y cada capítulo contiene ciertos artículos..." Lo explica en el prólogo (sin foliar, aunque posteriormente se le ha añadido con numeración romana el número 3 ).

16 Se extiende desde el folio 9 hasta el 16. Es de notar que aunque los folios están foliados con numeración romana, en el índice emplea la numeración arábiga para indicarnos dónde va a situar cada capítulo o tratado. Respetamos esta diferencia en nuestras citas a pie de página.

17 B. Caunedo del Potro y R. Córdoba de la Llave (2000) El Arte del Alguarismo..., pp. 68-72. El autor de este manuscrito nos habla también de siete especias, las operaciones aritméticas fundamentales. Para él son: suma, resta, multiplicación, división, reparto proporcional, regla de tres y fracciones. El sistema de numeración no constituye para él una espeçia, aunque le otorga una importancia singular, ya que es el punto de partida del arte del alguarismo. Vemos que hay también otras pequeñas diferencias con la regla de tres y las fracciones. La regla de tres es la sexta espeçia y allega espedaçados (fracciones), la séptima especia en el arte del alguarismo, no lo son en el tratado que aquí brevemente presentamos, en el que se explican como tratado independiente.

Las siete espeçias en el tratado de Mosén Juan de Andrés, se extienden a lo largo de los siguientes folios: nombrar, fols. 17 a 19; sumar fols. 19 a 25; restar fols. 26 al 30; multiplicar fols. 30 al 37; partir del 37 al 43; progresiones del 43 al 51 y raíces cuadradas y cúbicas fols. 57-56.

18 B.N. Raros, 9124, fols. 56 a 74.

19 B.N. Raros, 9124, Regla de tres, fols. 74 a 90 y regla de compañías, fols. 91 a 108. 
de dinero. Una barata con tiempo, tercer ejemplo que describe, contempla el caso en que uno de los mercaderes involucrado en la operación de trueque necesite tiempo para pagar y cerrar el trato. En todos los casos, ha de hallarse la solución para que ninguna de las partes salga perjudicada ${ }^{20}$.

En el séptimo tratado contempla la operación de cambio, en el que de nuevo, y tal y como nos tiene acostumbrados el autor, precediendo al conjunto de problemas -que va a resolver utilizando la regla de tresexplica su utilidad para los mercaderes, en este caso, para aquellos involucrados en operaciones de largo alcance. Lo hace de la siguiente manera... "has de saber que cambio real es aquel cambio que faze de una tierra en otra mediante una letra de cambio, la qual cosa se trata entre grandes mercaderes que tienen factores en muchas partes..."21.

Los problemas de aleaciones -que tampoco van a faltar en este tipo de manuales- los reúne y presenta en el tratado octavo, en el que también se preocupa por aclarar el término de aleación y el de quilate $^{22}$. Pospone hasta el tratado noveno ${ }^{23}$-que es el último en el que explica operaciones aritméticas-, ya que en el décimo ${ }^{24}$-presenta y resuelve diferentes problemas- la regla que denomina de la falsa posición ${ }^{25}$, que es la que le resulta más difícil de entender, y tiene que recurrir para probar su importancia a la autoridad de los clásicos. En realidad, en esta regla, que se acerca desde el punto de vista aritmético a las proporciones, se parte de uno o dos presupuestos falsos para hallar uno verdadero. Ello constituye la esencia de la regla, pero desconcierta enormemente a nuestro autor, le cuesta entenderlo y refuerza su explicación invocando la autoridad de Aristóteles ${ }^{26}$.

Después del índice y antes de desgranar con detalle las diferentes reglas anunciadas, se inserta una representación de los números

20 B.N. Raros, 9124, fols. 108 a 114.

21 B.N. Raros, 9124 , fols. 114 a 121

22 B.N. Raros, 9124 , fols. 121 a 128.

23 B.N. Raros, 9124 , fols. 128 a 138.

24 B.N. Raros, 9124, fols. 138 a 144

25 B.N. Raros, 9124, fol. CXXVIII v. "articulo primero de la definición de falsa posición y que quiere decir falsa posición y has de saber que falsa posición no quiere decir otra cosa en el arte mercantivol sino poner un número falsamente puesto o dos números falsamente puestos para fallar la verdad del número que queremos saber...".

26 B.N. Raros, 9124, fol. CXXIC r. "... no has de maravillar que por falso número o por falsos números se sale y se sabe la verdad. Car Aristóteles dixo, ex falsis verum ex vero non nisi verum. $Y$ así mismo dize el lógico que due negationes equivalent uni afirmationi". 
con los dedos y varias tablas de multiplicar, con la finalidad de facilitar su memorización.

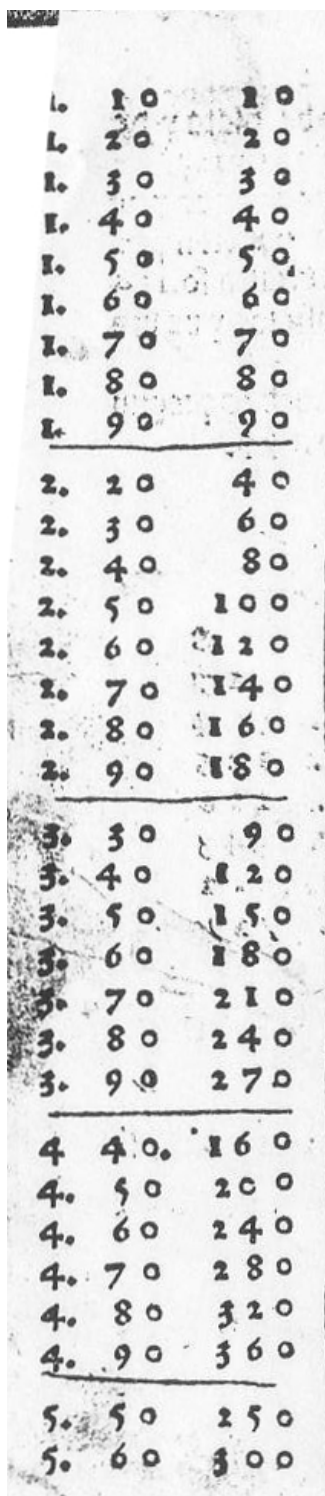

\section{La tabla majos.}

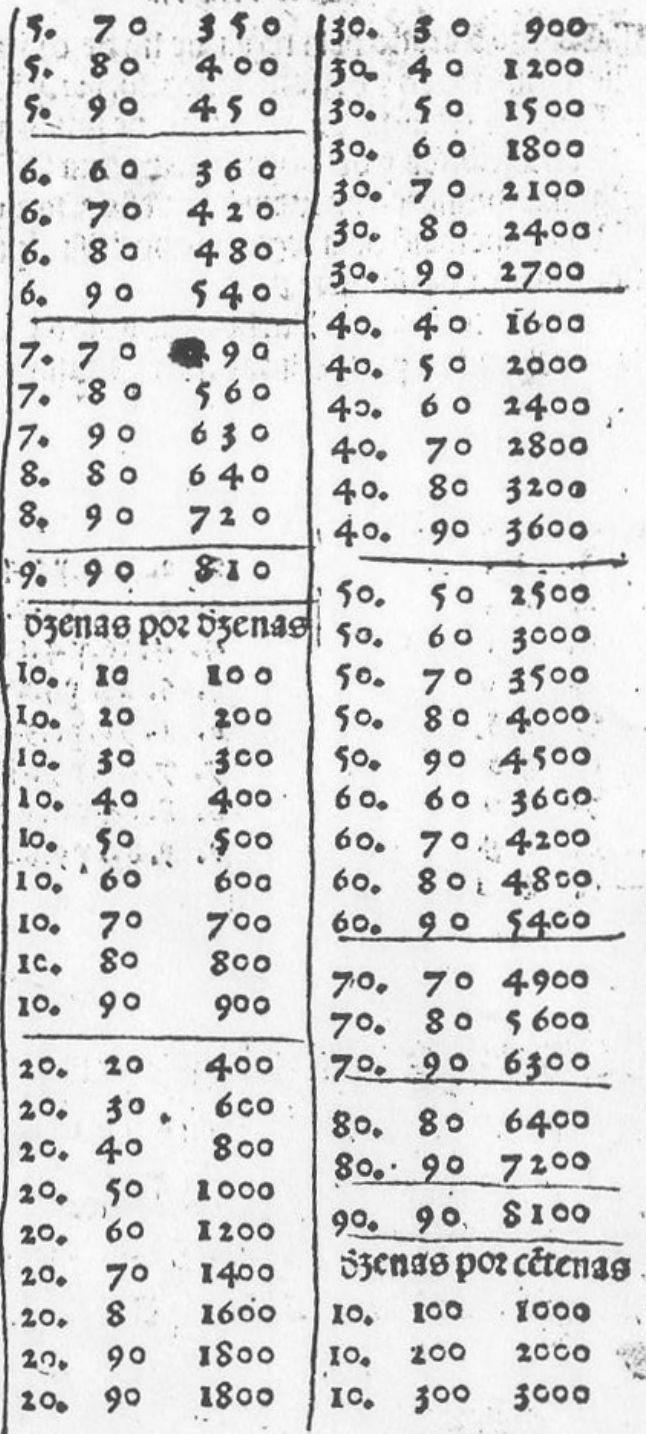




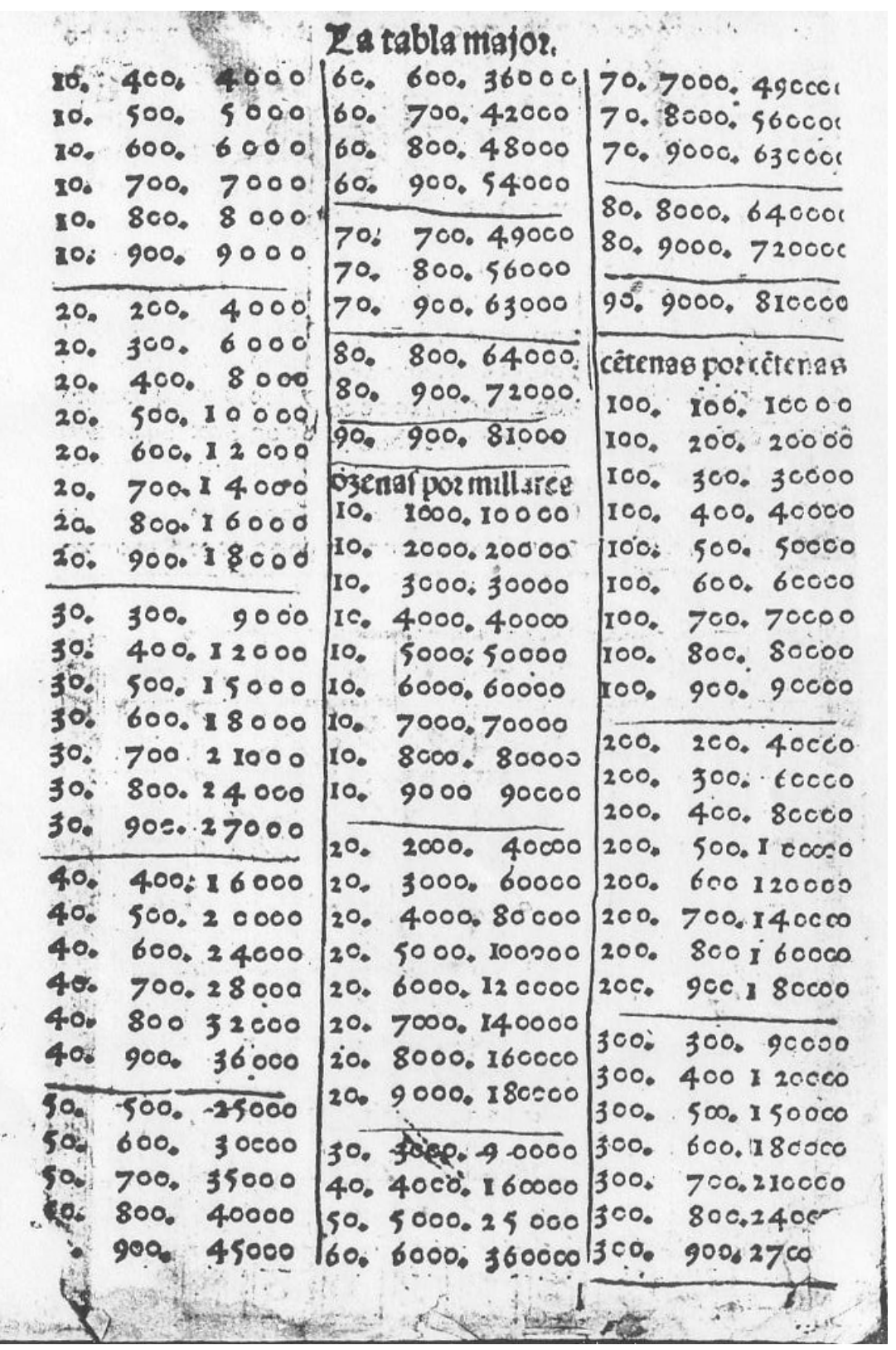

Tablas de multiplicar que el autor inserta para facilitar su memorización. Precede a sus explicaciones (Sin foliar).

Tras esta breve presentación general del trabajo, vamos a efectuar algunos comentarios y observaciones sobre el mismo, siguiendo el mismo orden del autor. 
El trabajo es una recopilación de conceptos y ejercicios que se desarrolla a lo largo de 10 extensos tratados en los cuales se definen en forma concisa y clara los procedimientos aritméticos necesarios para la resolución de los problemas que se plantearían a los mercaderes al realizar sus transacciones. Debemos destacar del mismo, no ya la introducción de nuevos conceptos -solamente nos facilita un procedimiento para conocer la suma de las proporciones- sino la perfecta agrupación y ordenación de los diversos problemas, así como su solución de una forma clara y evidente, que no siempre resulta breve.

\section{Primer Tratado 27}

El primer tratado sirve de introducción a la numeración y realiza una explicación de los números y sus propiedades tal y como se clasificaban en la época, pero no todos asimilables a las actuales. Si bien las descripciones de los números primos, pares, impares y compuestos corresponden exactamente al concepto actual, otros como los números superficiales, circulares o los triangulares, no son asimilables a la actualidad.

\section{Segundo Tratado ${ }^{28}$}

Ya entrando en las operaciones fundamentales, el segundo tratado -que resulta ser el más extenso- explica de manera amplia y detallada las siete especias, imprescindibles para todo mercader que quiera denominar este arte y que son: nombrar, sumar, restar, multiplicar, partir, progresiones, raíces cuadradas y cúbicas. Resulta fácil para el lector, ya desde la primera especia, nombrar, poder asimilar los conceptos de la numeración, ya que existe un continuo esfuerzo del autor por explicar no solamente el significado de los números y de su orden, sino también cualquier nuevo concepto que pueda resultar difícil en su interpretación. El sistema de numeración es ya evidentemente la numeración indoarábiga y se explica con claridad la notación posicional.

Muy extensas y acompañadas de ejemplos diversos resultan las páginas dedicadas a la suma y a la resta. Tras explicarnos en qué

\footnotetext{
27 B.N. Raros, 9124, fols. IX r. a XVII r.
}

28 B.N. Raros, 9124, fols. XVII v. a LVI v. 
consisten las operaciones, nos ofrece diversos ejemplos e incluye las ya tradicionales pruebas del 7 y del 9.

\begin{tabular}{l|lllll}
\hline Deue & 5 & 7 & 6 & 4 & oucados \\
Pago & 4 & 3 & 5 & 3 & oucados \\
\hline TRefta & 1 & 4 & 1 & $x$ & oucados \\
\hline PPrueus & 5 & 7 & 6 & 4 & oucados \\
\hline
\end{tabular}

Ejemplo de resta (Fol. XXVII r.)

Tras definir la multiplicación explica que hay diferentes formas de multiplicar, una que se llama de la ala, la más común entre los cristianos, y otra, multiplicar morisco, que es el modo y uso de los moros, y que el autor considera la más sana y la más segura. Su no uso lo atribuye exclusivamente a la ignorancia ${ }^{29}$, por lo que es muy prolijo en sus explicaciones. Éstas resultan claras en su procedimiento aunque largas a la hora del desarrollo de las operaciones.

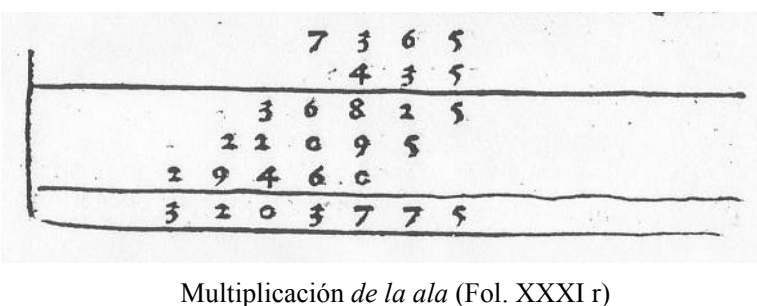

Analiza la división en el artículo tercero, donde también describe de un modo claro y sencillo cómo se realiza esta operación. Así lo aplica en sus primeros ejemplos, como el que corresponde al número 7368 partido por 3 , pero esta sencillez y claridad que se mantiene en los siguientes ejemplos desaparece cuando llega al capítulo siete en el que

29 "Has de saber que ay muchas maneras de multiplicar, pero dos maneras solamente quiero poner en este capitulo y la practica dellas y cómo se llama cada una manera. La primera se llama multiplicar de la ala y es la más común y usada multiplicación entre todos los mercaderes por todas partes de los cristianos y llámase de la ala por causa que fecha la operación y su suma quedará la figura de aquella operación así como una ala. La segunda manera se llama multiplicar morisco que es el modo y uso de los moros, la qual manera de multiplicar es la más sana y la más segura, en cara que entre los mercaderes crisitianos no se usa porque no la saben, ca si la supiesen otra manera no usasen..." B.N. Raros, 9124, fol. XXXI r. 
divide 83675 por 354 . En este caso, su interés por describir los más mínimos detalles se convierte en casi obsesivo y se alarga inútilmente en un tedioso procedimiento que consume varias páginas ${ }^{30}$.

Muy interesante y destacable resulta el capítulo sexto dedicado a la sexta especia, progresiones. En éste, además de las descripciones y ejemplos utilizados en la época, se debe destacar un paso muy importante, y que no habíamos detectado en los manuales medievales que conocemos, y es que el autor nos facilita el procedimiento para conocer la suma de las progresiones, y que resulta verdaderamente útil ${ }^{31}$. Como colofón a este interesante capítulo, una pequeña colección de problemas, ciertamente antiguos y conocidos en los tratados de aritmética medievales, como son los de caminantes que se encuentran o el de los caracoles que se cruzan recorriendo un ciprés, los cuales están perfectamente resueltos, basándose en sus conocimientos de las progresiones.
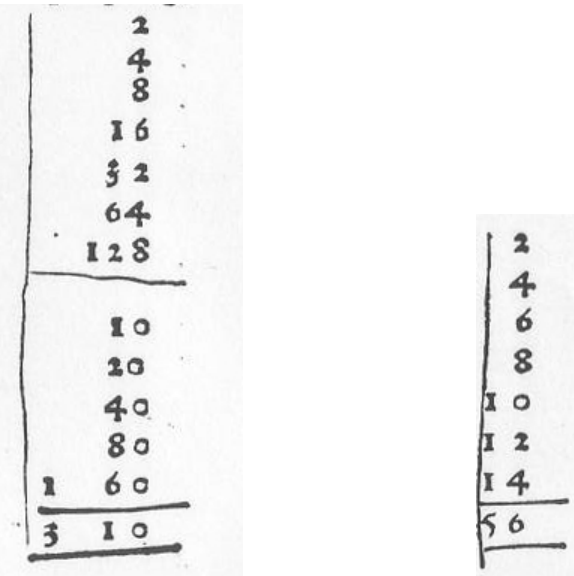

Progresiones (Fol. XLV v.)

30 Mientras que la definición de la operación y sus primeros ejemplos se extiende desde el folio XXXVII r. hasta el folio XLI v., el artículo siete se prolonga desde este fol. XLI v. hasta el XLIII r. Y solamente realiza una operación, 83675 dividido por 354.

31 También útil en la actualidad. En base a ello tenemos que decir, que si bien entonces se realizaba un procedimiento específico para calcular la suma de cada progresión, también es cierto que en la actualidad usamos procedimientos más generales, que con la misma fórmula podemos calcular la suma de muchas progresiones. Todo ello basado en que actualmente hacemos una división de las progresiones en aritméticas y geométricas, disponiendo de las herramientas matemáticas necesarias para el cálculo de las mismas. No obstante, resulta muy esclarecedor y práctico que el autor haya resuelto de manera sencilla y eficaz el cálculo de la suma de las progresiones el cual tiene total vigencia en nuestros días. 
Respecto a la séptima especia, extracción de raíces, podemos decir que sus ejemplos están bien resueltos, aunque con explicaciones que se alargan innecesariamente. Resuelve varias raíces cuadradas, raíces correspondientes a los quebrados, y también se atreve con ejercicios relativamente complejos como las raíces cúbicas, aunque ya nos anticipa antes de iniciar su resolución que es materia escabrosa y fuerte para entenderla y que además para el arte mercantivol muy poco es menester ${ }^{32}$.

\section{Tercer Tratado 33}

Operar con quebrados resultaba de suma utilidad en las transacciones mercantiles, de ahí que Juan de Andrés las presente en el tratado tercero con la importancia adecuada a su trascendencia. Con pulcritud define el quebrado y lo describe: "has de saber que el número que se pone encima de la raya se llama nombrador y el número que se pone debaxo de la raya se llama denominador y siempre debe ser el número que se pone encima de la raya menor que el numero que se pone debaxo"134. También clasifica todas las operaciones a realizar con los quebrados y dedica un capítulo a cada una de ellas: sumar, restar, multiplicar y dividir, reservando el último, el capítulo sexto, a la labor de encontrar denominador común. De la importancia de esta operación, nos deja constancia, cuando, por ejemplo, resuelve diferentes ejercicios en los que se nos invita a determinar cual de dos quebrados es mayor teniendo ambos distinto denominador ${ }^{35}$. Contempla, además, todos los casos prácticos posibles, es decir, operaciones con quebrados solamente, entero y quebrado con quebrado, así como la de entero y quebrado con entero y quebrado, en un intento de que no se escape ningún problema que le pueda surgir al mercader sin que haya sido tratado por el autor. Tampoco se olvida de las correspondientes pruebas de las operaciones que puedan verificar que todos los cálculos se realizaron correctamente ${ }^{36}$. Especial atención dedica a los problemas inversos en los que se nos pide, por ejemplo, calcular qué

32 B.N. Raros, 9124, fol. CIIII v. Nos permite comprobar que existió el conocimiento y la capacidad para realizar su cálculo, pero que no existía la necesidad de aplicación.

33 B.N. Raros, 9124, fols. LVI v. a LXXIIII r.

34 B.N. Raros, 9124, fol. LVII r.

35 B.N. Raros, 9124, fol. LXXI v.

36 El tratado tercero destinado a los quebrados y a sus operaciones es muy extenso. Se prolonga desde el fol. LVI v. hasta el fol. LXXIIII v. 
quebrado habría que añadir a uno determinado para que nos de un resultado conocido ${ }^{37}$.

\section{Cuarto tratado 38}

No podía faltar de ningún modo en un manual de aritmética mercantil, la sección correspondiente a la regla de tres. En palabras del autor, "tratado de la definición de la regla de 3 y de dónde procede tal regla y tiene tal fuerça que multiplicando el segundo por el terçero y partiendo por el primero se absolve y se sabe lo que queremos saber..."139. De esta manera concisa y pragmática define esta potente herramienta que en líneas generales mantiene su vigencia en la actualidad.

Dada la facilidad y sencillez de utilización de la regla de tres ha sido siempre considerada como una herramienta de extraordinario interés. El autor consciente de este alto valor para los mercaderes de la época -aparece en prácticamente todos los manuales medievales- hace un desarrollo detallado de la misma que culmina con una gran cantidad de problemas resueltos. Seis capítulos con importante contenido docente explican y aclaran cualquier duda que se pueda plantear a la aplicación de la regla de tres.

El primer capítulo empieza con la definición, describe a continuación cómo se resuelve la operación, para finalizar con la prueba de la misma.

Una vez explicada la operativa, que no reviste ninguna dificultad, ya nos adentramos en el capítulo segundo, que es una colección de 22 problemas diferentes perfectamente resueltos, dentro de la categoría establecida por el autor como regla de 3 sin tiempo. Se inicia el siguiente capítulo con una categoría superior de dificultad al añadir el factor tiempo a la regla de 3. Debemos añadir el tiempo al capital invertido ya que será un factor de vital importancia para el cálculo de la ganancia y que tendrá un valor directamente proporcional a dicho tiempo. Se describen siete problemas de forma detallada, encontrándose la correcta solución para los mismos.

\footnotetext{
37 Se especifican estos cálculos en B.N. Raros, 9124, fol. LXX v. - LXX r.

38 B.N. Raros, 9124, fol. LXXIIII v. a CXI r.

39 B.N. Raros, 9124, fol. LXXIIII v.
} 
El capítulo cuarto aporta el problema de los tantos por ciento, permitiendo calcular en cuanto se debe vender una mercancía para ganar un porcentaje determinado, así como el problema inverso, es decir, dado el precio de compra y venta calcular la ganancia en porcentaje. A lo largo de 16 problemas se realizan aplicaciones de la regla de 3 , en los cuales además de los cálculos correspondientes a ganancias, también se resuelven los correspondientes a casos de pérdidas.

Los problemas de regla de 3 en los que interviene renta y capital son descritos en el capítulo quinto y están desarrollados a lo largo de cinco problemas resueltos. Completa dicho capítulo un problema relativo a las aleaciones.

El último capítulo es un compendio de utilidades en las cuales el autor hace una generosa explicación de las equivalencias de monedas, peso y longitud. A lo largo de cinco artículos se describen las diversas equivalencias, reflejando las nada desdeñables diferencias entre las medidas de diversos lugares. Valga como ejemplo, que el ducado, en Aragón, vale 22 sueldos, en Valencia 21 sueldos y en Barcelona 24 sueldos, cuya máxima diferencia podemos establecer en un $14 \%$.

\section{Quinto Tratado ${ }^{40}$}

Que mejor definición de la regla de la compañía que la que nos ofrece Juan de Andrés: "trata de la operación que se faze entre dos o tres mercaderes y dende arriba en el arte mercantivol quando fazen compañía y ponen su canal diferentemente y sin tiempo y cómo debe ser la ganancia o pérdida entre los tales mercaderes..." ${ }^{41}$ en el capítulo primero del quinto tratado, ya que en el capítulo segundo hace referencia a las compañías con tiempo.

Dentro del apartado compañías sin tiempo, se describen trece problemas con todo lujo de detalles, en los que principalmente se calculan repartos de ganancias entre los mercaderes. Destacables el ya clásico problema en que uno de los mercaderes aporta una joya y se pide calcular el valor de la misma o el artículo dozeno en el cual se conocen las sumas de las aportaciones de varios de los mercaderes y se pide calcular la aportación de cada uno. Estos problemas, fáciles de resolver

40 B.N. Raros, 9124, fol. XCI r. a CVIII r.

41 B.N. Raros, 9124, fol. XCI r. 
en la actualidad por sistemas de ecuaciones, no lo son tanto sin disponer de estos recursos y denotan el ingenio y habilidad de los maestros de entonces para resolverlos de una manera relativamente sencilla y con perfecta validez.

Dentro del capítulo segundo, correspondiente a las compañías con tiempo, tenemos ocho problemas, que nos hacen ver de forma inexorable la importancia del factor tiempo en las operaciones mercantiles. Resulta de extraordinario interés la nueva valoración del tiempo que estos problemas claramente nos reflejan. Tras leer su enunciado, no se puede dudar que ya entonces el grupo mercantil sostenía firmemente que en cualquier operación con capital su rentabilidad dependía del tiempo. A lo largo de siete problemas se realizan los cálculos de las ganancias correspondientes a compañías con tiempo y se finaliza con un último problema en el que las aportaciones dinerarias se sustituyen por trabajo.

El capítulo tercero aporta un grado más de complejidad introduciendo una nueva variable, la del tanto por ciento, y realizando cálculos en los que se pueden producir ya tanto pérdidas como ganancias. En este tipo de problemas toma especial relevancia el factor y su incentivación ${ }^{42}$. Éste que es responsable de ejecutar las inversiones de capital, recibe a cambio de su trabajo, un tanto por ciento. No solamente es relevante por la introducción del factor propiamente dicho, sino porque además su existencia empieza a significar que las compañías que requieren sus servicios tienen especial importancia, alejándose claramente de la venta al detalle. De esta manera ocho problemas más nos abren las puertas del conocimiento de esta problemática, descubriendo la correcta solución a los mismos. En ellos se valora el trabajo del factor calculando su porcentaje en la ganancia, así como las ganancias que en ley corresponden a cada mercader por su aportación, teniendo lógicamente en cuenta el tiempo como valor ya inseparable a las aportaciones. De los ocho problemas descritos en este capítulo tercero, creemos que tienen especial interés los cuatro últimos en los que se producen incidencias en las aportaciones, tales como aportaciones extras realizadas posteriormente al pacto de la compañía o la introducción de un nuevo mercader a formar parte de la sociedad cuando ya estaban establecidas

42 Sobre la figura del factor en la Edad Media castellana, remito a mi trabajo (1998) "Factores burgaleses. ¿Privilegiados o postergados?", En la España Medieval, 21, pp. 97-113, y también a (2006) "La formación y educación del mercader", El Comercio en la Edad Media, XVI Semana de Estudios Medievales Nájera y Tricio, Nájera, Instituto de Estudios Riojanos, pp. 443-448. 
las aportaciones y ganancias de cada mercader. Estas nuevas incidencias agudizan el ingenio matemático del autor que debe afrontar una nueva situación en la compañía, el conseguir la equidad sin desvirtuar los criterios de reparto de la firma inicialmente constituida.

El capítulo cuarto relativo a preguntas, que se resuelven por la regla de la compañía, nos lleva inequívocamente a recordar que estos problemas eran ya clásicos muchos años atrás. Problemas de herencias complicadas con el nacimiento de mellizos, los de compañía para pacer los ganados del mismo yerbaje o los del pastor que cuida las ovejas a cambio de una determinada ganancia ${ }^{43}$. La ingenuidad de sus enunciados y su acercamiento a situaciones de la vida diaria, nos muestran una vez más el carácter práctico tantas veces comentado de este tipo de obras.

\section{Sexto Tratado ${ }^{44}$}

Se refiere el siguiente tratado al arte de conseguir la equidad en el intercambio de mercancías cuando los mercaderes tratan de introducir un sobreprecio al valor de las mismas. Existen tres clases de baratas: la barata simple en la cual sólo se intercambian mercancías, la barata compuesta en la cual además de mercancías alguno de los mercaderes aporta dinero y la tercera barata con tiempo en la cual alguno de los mercaderes pide tiempo para pagar.

Evidentemente en la colección de problemas que siguen a la descripción de las baratas, se da el caso, que intentando un mercader compensar el posible sobreprecio que impone el otro, no lo consigue y resulta engañado. Se pide que se realice el cálculo de quien ha salido engañado y en cuanto. En general, sin embargo, el objetivo de los problemas es conseguir que la barata sea lícita.

En el capítulo de la barata compuesta, la mayor parte de los problemas corresponden a mercaderes que solicitan en el intercambio una parte en dinero, posibilidad que se suele expresar como una fracción en el valor de la mercancía, aunque también se da el caso de querer ganar un tanto por ciento en la transacción, como es el caso del artículo quinto.

43 Ver ejercicios análogos por ejemplo, B. Caunedo del Potro y R. Córdoba de la Llave (2000) El Arte del Alguarismo. Un libro castellano de aritmética comercial y de ensayo de moneda del siglo XIV. Salamanca: Junta de Castilla y León, pp. 19-20.

44 B.N. Raros, 9124, fol. CVIII r. a CXIII v. 
En lo referente a baratas con tiempo, se añade este importante factor a la transacción, que ya no solamente afecta al valor de la mercancía ya que el tiempo multiplica al mismo, sino que además se convierte en incógnita del problema, a tener en cuenta durante el desarrollo de los cálculos mismos. Así, en el artículo cuarto, en que la cuestión a resolver es demando a que tiempo se debe meter para que la barata sea igual... ${ }^{45}$.

\section{Séptimo Tratado ${ }^{46}$}

Importante tratado dedicado al cambio y que es definido por el autor en la siguiente expresión: "cambio real es aquel cambio que faze de una tierra en otra mediante una letra de cambio, la cual cosa se trata entre grandes mercaderes que tienen factores en muchas partes..." 47. Tenemos que resaltar aquí la importancia de las nuevas circunstancias y valores que se desprenden de esta definición. Hay que trasladar evidentemente el valor de las mercancías de un lugar a otro, y calcular la equivalencia de monedas entre las mismas. Además aparece la figura de la letra de cambio como valor que requerirá un pago, pasando ineludiblemente por el factor ya que las transacciones no se realizan en el mismo tiempo y lugar. Evidentemente eran éstas circunstancias que atañían a las grandes firmas que operaban en el que podemos denominar mercado internacional. El autor es claro al respecto la cual cosa se trata entre grandes mercaderes que tienen factores en muchas partes...

Dada la variedad de monedas utilizadas en los cambios y la movilidad de los mismos, no resulta raro en algunos problemas, comprobar que un determinado mercader quisiese recibir el dinero en distintas monedas como aparece en el artículo cuarto del capítulo segundo en que el mercader solicita ser pagado tanto en moneda valenciana como aragonesa. En el siguiente artículo, el quinto, nos muestra como un mercader que posee ducados viejos y nuevos, va a un cambiador para obtener libras. Lógicamente los ducados tendrán un valor distinto.

\footnotetext{
45 B.N. Raros, 9124, fol. CXII v. - CXIII r.

46 B.N. Raros, 9124, fol. CXIII v. a CXXI v.

47 B.N. Raros, 9124, fol. CXIII v. Creemos que hay un pequeño error en la foliación, ya que se lee CXIIII v.
} 
Ya en el tercer capítulo, el autor trata de resumir con un buen número de ejemplos las reducciones de monedas. Una colección de diez problemas desarrollados en este capítulo, conforman las posibles situaciones a las que se puede enfrentar el mercader para realizar cambios y describen de manera concisa la equivalencia que es necesario conocer para realizar las correctas transacciones.

\section{Octavo Tratado ${ }^{48}$}

Interesante tratado aunque quizá un poco corto, que trata del fin de oro y plata que se define: "quiere decir oro de tantos quilates y plata de tantos dineros de ley y del peso del oro y de la plata y quántas maneras ay de fin de oro y de plata..." 49 .

No resulta raro por otra parte, que el autor sólo haya dedicado una pequeña parte del trabajo al tema de las aleaciones, ya que analizado en profundidad, es un tema tan extenso que permite la dedicación de manuales exclusivos. Tenemos buenos ejemplos de ellos ${ }^{50}$, por lo que, sin duda, el autor decidió dar una pequeña prueba de la existencia de las aleaciones, pero sin adentrarse en un tema que es realmente específico y profundo en cuando su análisis.

Se inicia el tratado con los dos primeros artículos en los que se define lo que quiere decir el fin de oro y plata, ya en sus palabras técnicas correspondientes del quilate en el oro y dinero de ley en la plata, que es lo que conocemos también como pureza de estos metales preciosos.

Se desarrollan diversos problemas en los cuales si que se describe la problemática de las aleaciones de forma clara y se transmite al lector las diversas situaciones que se pueden plantear, desde el problema más elemental consistente en aumentar o reducir la ley de una aleación,

48 B.N. Raros, 9124, fol. CXXI v. a fol. CXXVIII v.

49 B.N. Raros, 9124, fol. CXXI v.

50 El trabajo ya citado de B. Caunedo del Potro y R. Córdoba de la Llave (2000) El Arte del Alguarismo..., ed. cit., ofrece además de la edición y estudio del primer tratado conocido de aritmética mercantil castellano el de ensayo de moneda bajo el título "Cálculo, Técnica y Moneda. Un Manual castellano de ensayadores y maestros de moneda del siglo XIV", que corre a cargo de Ricardo Córdoba de la Llave. Yo misma he individualizado su problemática en "Three Castilian Manuscripts on Mercantile Arithmetic: Their problems of alloys", presentada en el Coloquio Culture monétaire, aspects mathématiques, technologiques et marchands (XIII-XVI siécle),celebrado en París y Orleans en Septiembre del 2004 (actas en prensa). 
hasta la que pueda resultar más compleja de conocer la ley de una mezcla de aleaciones.

En cuanto a los tipos de aleaciones, también se hace la distinción, de aquellas en las que se utilizan metales adicionales para rebajar la ley del metal precioso. El artículo cuarto del capítulo $4^{\circ}$ nos demanda tácitamente ¿quantas arrobas de alambre tienen las 10 arrobas?... siendo el inicio del ejercicio... yo tengo 10 arrobas de oro de 18 quilates. Demando ¿quantas arrobas de alambre tienen las 10 arrobas? $?^{51}$. El artículo dezeno ya refiriéndose a la adición del alambre nos dice... añadiendo quatro onzas de alambre tornó de 8 dineros de ley... Yo tengo argento de 11 dineros de ley y no sé quantas onzas hay, pero añadiendoquatro onzas de alambre tornó de 8 dineros de ley. Demando ¿quántas onzas eran de primero $?^{52}$.

Queda bien claro, pues, que si bien no es extenso el desarrollo de este tratado, si resulta plenamente didáctico, en cuanto a que son planteados de manera explícita, los problemas más importantes de la amplia gama que son las aleaciones.

\section{Noveno Tratado ${ }^{53}$}

Hace referencia este tratado a las reglas de las falsas posiciones definida como: "y has de saber que falsa posición no quiere decir otra cosa en el arte mercantivol sino poner un número falsamente puesto o dos números falsamente puestos por fallar la verdad del número que queremos saber ${ }^{\text {"54 }}$. En términos actuales diríamos que las reglas de las falsas posiciones equivaldrían a poner uno o dos números de prueba, que tienen relación con el resultado y a través de sencillas operaciones llegar al mismo.

Aunque la validez matemática de las reglas de las falsas posiciones es evidente y los resultados son correctos, actualmente resolvemos este tipo de problemas mediante sistemas de ecuaciones, los cuales son más intuitivos y rápidos. Juan de Andrés recurre en su presentación al argumento de autoridad. En un primer momento, en el

\footnotetext{
51 B.N. Raros, 9124, fol. CXXVI v.

52 B.N. Raros, 9124, fol. CXXVIII r.

53 B.N. Raros, 9124, fol. CXXVIII v. a CXXXXVIII v.

54 B.N. Raros, 9124, fol. CXXVIII v.
} 
índice, invocaba como hemos visto a Aristóteles, ahora, en el cierre del tratado a Luca Paccioli ${ }^{55}$.

El capítulo segundo del tratado, desarrolla una colección de problemas basados generalmente en cálculos con números quebrados, en los cuales el número de la falsa posición es múltiplo común de los denominadores, que elegimos nosotros y que lógicamente tiene que ver con el resultado al que llegamos mediante sencillas operaciones. Caso especial es el del artículo cuarto del capítulo tercero, en el cual se hace uso de raíces cuadradas para solución del mismo.

En el capítulo cuarto de este tratado, relativo a preguntas y cuestiones, se desarrollan tres problemas que se resuelven mediante la regla de las dos falsas posiciones. Se utilizan en ellos gráficos altamente descriptivos de los resultados a los que llegamos con los dos números falsamente puestos, y del análisis de las soluciones a las que llegamos podemos ver el error producido y así deducir el resultado correcto.

Así pues, como decíamos al principio, lo que llaman dos números falsamente puestos, traducido al lenguaje actual serían dos números de prueba, los cuales producen un resultado erróneo, pero éste comparado con el resultado verdadero nos permitirá llegar a la solución del problema tal como indicábamos inicialmente.

\section{Décimo Tratado ${ }^{56}$}

Este último y corto tratado dedicado a: "preguntas y cuestiones que son necesarias de saber en el arte mercantivol"57 tiene gran importancia ya que añade al desarrollo de los problemas un factor importante y decisivo para su solución como es el análisis del mismo. Las matemáticas con ser una ciencia exacta, no significan de ninguna manera que la metodología empleada para la solución de un problema tenga que ser siempre la misma, sino muy al contrario, del análisis del problema antes de iniciar su resolución, dependerá en gran parte la facilidad y rapidez de su solución.

55 "Nota y has de saber que estas reglas suso declaradas de posición tienen la fuerça de la proporción y proporcionalidad según lo pone Lucas de Burgo en su tratado mayor // la qual sería muy superflua de ponerla en este breve tratado", B.N. Raros, 9124, fol. CXXVII v. CXXXXVIII $r$.

56 B.N. Raros, 9124, fol. CXXXXVIII r. a CXLII v.

57 B.N. Raros, 9124, fol. CXXXX r. 
Empieza el tratado con un problema resuelto por la regla de las dos falsas posiciones, pero en el segundo, el autor nos plantea acertadamente, que aunque dicho problema podría ser resuelto como el anterior por la regla de dos falsas posiciones, nos afirma que también se puede hacer por la regla de tres, muy fácil y sotilmente. A lo largo de los siguientes ejercicios desarrollados en este último tratado, el autor nos confirma que el análisis del problema es vital para su pronta solución y demuestra que no siempre el método convencional es el mejor para resolverlo. Hace alarde de la facilidad con que podemos llegar al resultado utilizando en cada caso el procedimiento idóneo para su solución.

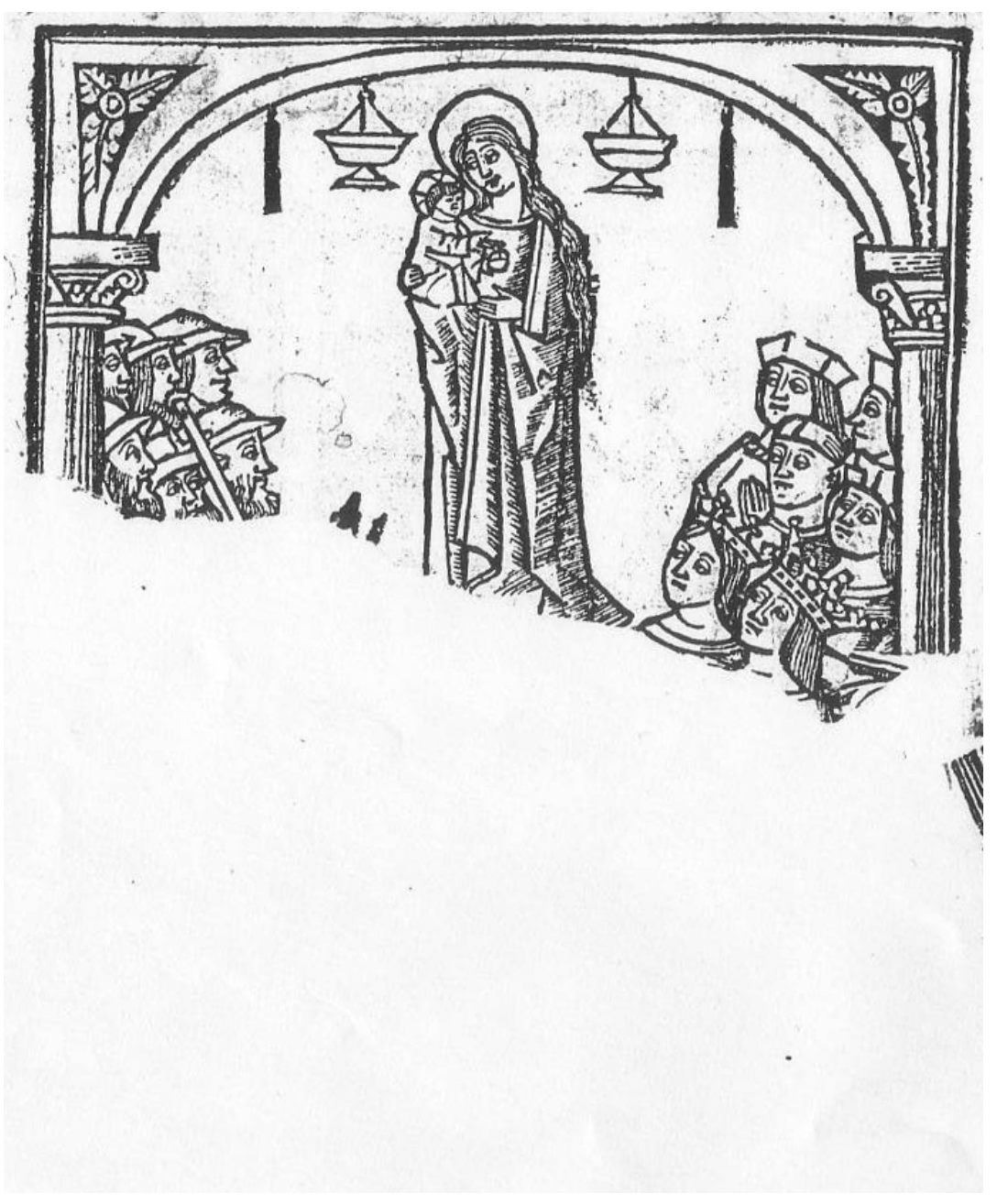

Inicio del Manual 


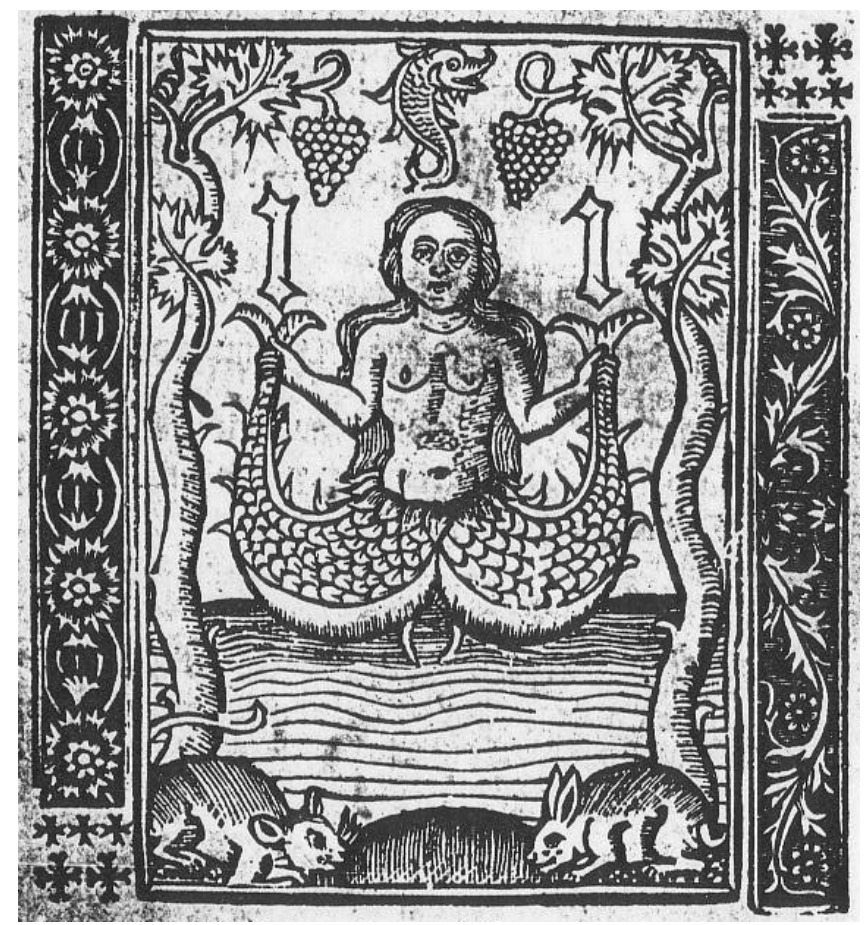

Ilustración con la que se concluye la obra

\section{BIBLIOGRAFÍA}

CaSAdo Alonso, H. (1999) "Comercio y nacimiento del Estado Moderno en Castilla (S. XIV - XVI). Algunas reflexiones a la luz de nuevas corrientes de investigación internacional", El Estado en la Baja Edad Media. Nuevas perspectivas metodológicas. Universidad de Zaragoza, pp. 51-75.

CASTILlo GómeZ, A. (1997) De Escrituras y Escribientes. Práctica de la cultura escrita en una ciudad del Renacimiento. Las Palmas de Gran Canaria: Fundación de Enseñanza Superior a distancia.

CAUNEdo DEL POTRO, B. (1998) "Factores burgaleses. ¿Privilegiados o postergados?", La España Medieval, 21, pp. 97-113.

- (2003-04) "De Arismética. Un manual de aritmética para mercaderes", Cuadernos de Historia de España, LXXVIII, pp. 35-46.

- (2006) "La formación y educación del mercader", El Comercio en la Edad Media, XVI Semana de Estudios Medievales Nájera y Tricio, Nájera, Instituto de Estudios Riojanos, pp. 417-454. 
- y CóRdoba de la LlaVe, R. (2000) El Arte del Alguarismo. Un libro castellano de aritmética comercial y de ensayo de moneda del siglo $X I V$. Salamanca: Junta de Castilla y León.

- y - (2004) "Oficios Urbanos y desarrollo de la Ciencia y de la Técnica en la Baja Edad media: La Corona de Castilla", Norba, Revista de Historia, 17, pp. 41-68.

Certaldo, P. (1945) Libri di Buoni Customi, a. cura di A. Shiaffini. Firenze: F. Le Monnier.

Cifuentes I Comamala, LL. (2002) La Ciencia en cátala a l'Edad Mitjana i el Renaixement. Barcelona: Universitat de Barcelona.

CIPolla, C. (1994) El gobierno de la moneda. Ensayos de historia monetaria. Barcelona: Crítica.

Gallardo, J. (1968) Ensayo de una biblioteca española de libros raros y curiosos. Madrid: Gredos.

Gual Camarena, M. (1981) El primer Manual Hispánico de Mercaderia (siglo XIV). Barcelona: Consejo Superior de Investigaciones Científicas.

HeRnÁNDEZ ESTEVE, E. (1981) Contribución al estudio de la historiografía contable en España. Madrid: Banco de España.

HoYRuP, J. (2005) "Leonardo Fibonacci and Abbaco culture: a Proposal to Invert the Roles", Revue d'Histoire des Mathématiques, 11, pp. 23-56.

MALET, A. (ed.) (1999) Summa de lárt d'Aritmética de Francesc Sabtcliment. Vich: Eumo.

MARTIN ABAD, J. (2001) Post incunables ibéricos Julián Martín Abad. Madrid: Ollero \& Ramos.

- (2003) Los primeros tiempos de la imprenta en España (c. 1471 - 1520). Madrid: Ed. Del Laberinto.

SANTAMARÍA ARÁNDEZ, A. (1993) "La compilación mallorquina de mercaderia de Domingo Pau", Homenatge a Antoni Mut Calafell, arxiver. Mallorca: Govern Balear, pp. 249-276.

SeVILlano Colón, F. (1974-79) "Un Manual mallorquín de mercaduria medieval", Anuario de Estudios Medievales, 9, pp. 517-530.

SIGLER, L.E. (2002) Fibonacci's Liber Abaci. A Translation Into Modern English of Leonardo Pisano's Book of Calculation. New York: Springer.

van EGMOND, W. (1980) Practical Mathematics in the Italian Renaissance: A catalog of Italian Abbacus Manuscripts and Printed Books to 1660. Firenze: Istituto e Museo di Storia della Scienza. 